\title{
Desenvolvimento de um Modelo de Mobilidade Urbana em Tempo Real para Simuladores de Rede
}

\author{
Matheus Sanches Quessada $^{1}$, André Luis Cristiani ${ }^{1}$, Pedro Luís Ranzani Júnior ${ }^{1}$, \\ Rickson Simioni Pereira ${ }^{1}$, Matheus Pereira Leal ${ }^{1}$ e Rodolfo Ipolito Meneguette ${ }^{1}$ \\ ${ }^{1}$ Instituto Federal de São Paulo (IFSP) - Catanduva \\ \{matheus.quessada, andre.cristiani, pedro.ranzani\}@aluno.ifsp.edu.br
}

\begin{abstract}
Simulation is a more adopted approach to be feasible vehicular technologies, since they allow the existence of new protocols and infrastructures in a complete way, that is, what is necessary in all possible scenarios. For the simulations to be a necessary boost, the simulation environment of an environment must be real. Therefore, both the network parameters and the mobility model must represent a real network topology with high frequency, that is to say, in addition to requiring the network to match the parameters and mechanisms of the real ones, the action model also has represent real-world mobility. With this, a machine was proposed that collects the vehicle route, in real time, of a city that controls the data in its simulation environment.
\end{abstract}

Resumo. Simulação é a abordagem mais adotada para avaliar redes veiculares, pois permite avaliar novos protocolos e infraesturutras de forma completa, ou seja, avaliar novas ferramentas em todos os cenários possiveis. Para que essas simulações possam obter um resultado confiável é necessário que o ambiente de simulação utilizado aproxime-se de um ambiente real. Portanto, tanto os parâmetros de rede, quanto o modelo de mobilidade têm que representar a topologia de rede real com alta fidelidade, isto é, além de exigir que parâmetros de rede coincidam com os parâmetros e comportamentos dos equipamentos reais, o modelo de mobilidade também tem que representar a mobilidade do mundo real. Com isso, foi proposta uma ferramenta que coleta o percurso dos veículos, em tempo real, de uma cidade que permitirá ao pesquisador utilizar esses dados em seu ambiente de simulação.

\section{Introdução}

Simulação é a abordagem mais adotada para avaliar soluções de redes veiculares [Grzybek et al. 2012, Boukerche and Meneguette 2017]. A avaliação de desempenho é um importante desafio enfrentado por investigadores, dadas as características particulares deste tipo de rede, como uma rede altamente dinâmica e de grande topologia de escala. A condução de experimentos reais, usando veículos comuns, é muito cara e demorada, assim como sua abordagem, particularmente quando uma avaliação em larga escala é contratada. Além disso, não há público disponível em grande escala de testes. É improvável que um teste de larga escala esteja disponível no futuro próximo devido a difícil implantação e aos custos de manutenção. Simulação, por outro lado, é uma abordagem custo-efetiva e de larga escala, amplamente adotada por pesquisadores [I. Meneguette et al. 2018]. No entanto, a confiabilidade da simulação e dos resultados dependem de mecanismos veiculares apropriados e precisos, de alta capacidade para representar a topologia de rede com fidelidade.

De acordo com a taxonomia elaborada por Harri e colaboradores [Harri et al. 2009], os modelos de mobilidade podem ser classificados como: rastros (traces), baseados em pesquisas de aplicações reais e sintéticos gerados por simuladores de tráfego e mobilidade urbana. Rastreamentos de mobilidade veicular tipicamente descrevem a posição dos veículos ao longo do tempo e são usados para trazer realismo as ferramentas de simulação. Com o avanço dos dispositivos habilitados para GPS, trajetos reais coletados pelos veículos, durante sua rotina diária, estão disponíveis publicamente na literatura [Bracciale et al. 2014,Huang et al. 2007, Meneguette and Boukerche 2017]. Essas trajetórias reais são muito úteis para o processo de avaliação, pois definem movimentos 
de veículos reais e são utilizadas em diferentes cenários. Entretanto, as redes veiculares ainda não são utilizadas em larga escala, pois existe dificuldade em obter trajetos reais.

Muitos trabalhos utilizam os simuladores de trânsito para a geração de traces de mobilidade, que são usados como entrada em simuladores de redes. Esses dados já foram utilizados para experimentos com protocolos [Wang et al. 2015] e aplicações [Kuran et al. 2015] em VANETs. Entretanto, a maioria dos rastros gerados possuem um pequeno número de veiculos e foram simulados em cidades de pequeno e médio porte na Alemanha [Uppoor et al. 2014] e Luxemburgo [Codeca et al. 2015].

Com o objetivo de prover um serviço de rastreamento em tempo real, considerando não somente ônibus e carros, mas também toda a comunidade de uma cidade, como a cidade de Catanduva, em São Paulo, este trabalho propõe gerar uma base de dados com trajetos reais, considerando todos os aspectos da cidade para serem utilizados em simuladores de redes, tendo como objetivo trazer a simulação a um ambiente mais próximo do real, permitindo ao pesquisador que gere o seu trace de mobilidade no momento em que ele achar mais adequado. Essa solução também permitirá o monitoramento do tráfego da cidade de Catanduva, considerando todos os veículos que trafegam em suas vias. A captura da mobilidade do tráfego da cidade será gerada por um aplicativo móvel, e uma vez que essa informação esteja na ferramenta, o pesquisador poderá selecionar o intervalo do dia e a hora nos quais ele queira gerar a simulação.

O restante deste artigo está organizado da seguinte forma: na próxima seção, são mostradas técnicas de trajetórias e alguns trabalhos os quais apresentam trajetos reais; a ferramenta proposta de rastreamento de veículos em tempo real para a geração de traces para simulação de rede está descrita na seção 3; uma pequena avaliação do desempenho do aplicativo desenvolvido e de seus resultados são apresentados na seção 4; e, por final, a seção 5 apresenta as conclusões.

\section{Trabalhos Relacionados}

Os trajetos reais de mobilidade são aqueles gerados por veículos reais, equipados com dispositivos habilitados para GPS. Normalmente, os traços reais de mobilidade representam a mobilidade dos táxis, já que é mais fácil implantar e manter esse tipo de experimento em veículos dessa categoria do que em veículos comuns. Entre os vestígios reais de mobilidade existentes na literatura, selecionamos três para serem utilizados neste trabalho: Roma, São Francisco e Xangai. A seleção foi motivada pela alta demanda desses dados e suas localizações geográficas que representam três partes diferentes do mundo, sendo essas Europa, América do Norte e Ásia.

Roma [Bracciale et al. 2014] contém a posição dos táxis que trabalharam durante todo o mês de fevereiro de 2014. Cada motorista de táxi tem um dispositivo Android e está equipado com um receptor GPS que periodicamente recupera sua posição e envia para um servidor central. Posições com erros de precisão acima de 20 (vinte) metros foram ignorados. Este rastreio contém, por todo o mês, um total de 21.817 .851 (vinte e um milhões oitocentos e dezessete mil oitocentos e cinquenta e um) posições de 316 (trezentos e dezesseis) táxis. Em média, cada veículo contribuiu com 69.040 (sessenta e nove mil e quarenta) posições para todo o período de coleta. Contudo, poucos veículos contribuíram com valores mais altos até 118.500 (cento e dezoito mil e quinhentos), enquanto outros contribuiram com valores tão baixos quanto 19 (dezenove) posições.

São Francisco [Piorkowski et al. 2009] contém entradas de posição de 536 (quinhentos e trinta e seis) táxis que trabalharam durante o mês de maio de 2008. Cada táxi tem um receptor GPS instalado e envia informações de localização (identificador, carimbo de data/hora, latitude, longitude) periodicamente para um servidor central. Este rastreio contém, para o mês inteiro, um total de 11.219.955 (onze milhões duzentos de dezenove mil novecentas e ciquenta e cinco) entradas de posição. Cada táxi contribuiu, em média, com cerca de 20.930 (vinte mil novecentas e trinta) entradas. Poucos destes veículos contribuíram com valores significativamente menores, enquanto outros contribuíram com medidas mais altas de até 49.370 (quarenta e nove mil trezentas e setenta).

Xangai [Huang et al. 2012] apresenta posições de 4.316 (quatro mil e trezentos e dezesseis) táxis de fevereiro a abril de 2007. No entanto, podemos encontrar publi- 
camente disponíveis apenas os dados de um único dia de fevereiro, contendo 6.075.587 (seis milhões setenta e cinco mil quinhentos e oitenta e sete) entradas de posições. Semelhante aos outros casos, os táxis eram equipados com dispositivos habilitados para GPS, que enviavam suas informações de posição, periodicamente, para um servidor. Em média, cada veículo coletou cerca de 1.408 (mil quatrocentos e oito) inscrições para o único dia disponível publicamente. Poucos veículos contribuiram com valores menores, enquanto outros contribuiram com valores mais elevados, de até 7.011 (sete mil e onze).

Embora os exemplos apresentados descrevam trajetórias reais, a maioria foi criada utilizando uma quantidade pequena de veículos, coletando o rastreamento apenas de táxis. Além disso, esses traces foram capturados apenas em alguns dias. A ferramenta proposta utiliza o smartphone dos condutores dos veículos, sendo estes condutores responsáveis por informar o tipo de veículo sendo utilizado, podendo ser carro, moto, ônibus, entre outros tipos de veículos. Portanto, neste trabalho, ao contrário dos demais, são utilizados diversos tipos de veículos para monitorar e gerar rastreamento de veículos dentro da cidade de Catanduva. Além disso, a infra-estrutura proposta fornece uma captura em tempo real das informações de mobilidade do veículo, trazendo um cenário mais confiável para a simulação de rede, permitindo ao pequisador selecionar o melhor dia e horário para sua captura.

\section{Desenvolvimento do Modelo de Mobilidade Urbana em tempo real para Simuladores de Rede}

Esta seção descreve a ferramenta proposta, na qual provê uma interface para rastreamento de veículos de uma cidade, que poderá ser utilizada em simuladores de rede. Além de prover os arquivos de mobilidade em tempo real, necessários para os simuladores de rede, MobUrb tambem proverá informações suficientes para a agregação de novos serviços de roteamento de veículos e/ou busca de rotas otimizadas, tanto para os cidadãos, quanto para os condutores de veículos de preparação e resgate de emergências ou outros órgãos que auxiliam na segurança da cidade. $\mathrm{O}$ aplicativo captura as informações do percurso de seus usuários, de maneira totalmente anônima, armazenando os seguintes dados: tipo de veículo do usuário, podendo ser: carro, moto, bicicleta, ônibus, caminhão ou não informar, caso o usuário prefira; a data e hora inicial e final do percurso; a média de velocidade em que o usuário trafegou e as coordenadas de seu percurso, juntamente com a data e hora em que foram coletadas. Portanto, a ferramenta proposta usa serviços móveis de mapeamento geográfico para a captura das informações de coordenadas do veículo de tempos em tempos, sendo esse tempo correspondente ao deslocamento do veículo nas vias. Essas informações são transmitidas para uma central de controle responsável por monitorar o fluxo de veículos em uma cidade, bem como prover um trace de mobilidade da cidade, que poderá ser utilizado em simuladores de rede.

Esta ferramenta é composta por três módulos:

- Cloud: contém todo o armazenamento e processamento das informações, essas funcionalidades são providas pelo Firebase Realtime Database.

- Aplicativo de Sensoriamento: fica instalado nos celulares e é responsável por capturar as coordenadas dos percursos dos usuários e encaminhar para o banco de dados.

- Website: é acessível por meio da web e é reponsável por prover um monitoramento, em tempo real, dos usuários que estão utilizando o aplicativo. É, por meio do website, pode-se obter as informações contidas no banco de dados e a geração das informações de mobilidade para os simuladores de rede.

\subsection{Cloud}

Para o desenvolvimento dos serviços de nuvem, foi utilizado o banco de dados Firebase, que oferece diversas funcionalidades e infraestrutura suficiente para conexão e comunicação de diferentes tipos de dispositivos. O Firebase é um tipo de ferramenta que oferece o backend como serviço (BaaS), proporcionando, para os desenvolvedores, uma maneira simplificada de configurar e operar um backend em cloud para aplicativos móveis e web. Esta ferramenta foi escolhida pela escalabilidade e heterogenidade que 


\section{Tabela 1. Atributos armazenados no banco de dados}

\begin{tabular}{|c|c|} 
Parâmetro & Valor \\
Id veículo & 3 \\
Tipo de veículo & Veículo \\
Data & $21 / 03 / 2019$ \\
Hora & $12: 23$ \\
Latitude & 252 \\
Longitude & 1225
\end{tabular}

proporciona para os dispositivos que consomem seus serviços.O serviço utilizado foi o Firebase Realtime Database, um banco de dados não relacional hospedado na nuvem. Sua estrutura de armazenamento é baseado em JSON (Javascript Object Notation) e seus dados são sincronizados em tempo real com os clientes que se encontram conectados. Esses dados permanecem disponíveis mesmo quando a aplicação está off-line. Os atributos armazenados no banco de dados estão descritos na Tabela 1.

\subsection{Aplicativo de Sensoriamento}

Para a construção da aplicação foi utilizada a linguagem Java, através da IDE do Android Studio 3.0. O aplicativo possui apenas uma interface, onde é possível realizar as ações e não há necessidade de possuir uma conta de acesso ou realizar login para acessá-la.

O aplicativo necessita de duas permissões do usuário para ser utilizado, ambas são responsáveis por obter a localização do mesmo. A primeira forma de obter a localização é utilizando o GPS do próprio celular, que possui melhor desempenho e precisão ao identificar a localização do usuário. Esse método utiliza o GPS do celular para se comunicar com satélites e adquirir a localização atual. A segunda forma é acionada quando ocorre algum problema que causa indisponibilidade na primeira. Esta forma obtém a localização aproximada do aparelho por meio da conexão com a internet. No entanto, além de ter uma menor precisão, essa forma aumenta o consumo de dados do aparelho. A Figura 1 mostra a tela principal que possui as 3 (três) ações possíveis no aplicativo, que são: iniciar o serviço, parar o serviço e sair do aplicativo.

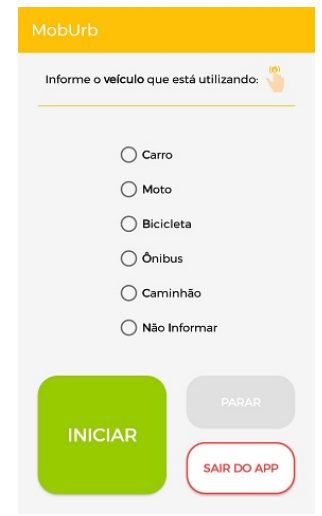

Figura 1. Aplicativo de Sensoriamento

Ao clicar no botão iniciar, o serviço de captura de localização é iniciado e o aplicativo é colocado em segudo plano, permitindo que o usuário execute outros aplicativos e atividades em seu smartphone. A cada 3 (três) segundos, o aplicativo solicita a localização do usuário, onde são obtidas as coordenadas da posição atual e a velocidade de movimento, que serão utilizadas para calcular a média de velocidade durante todo o percurso que o usuário realizou. Ao clicar em parar ou sair do aplicativo, o serviço de coleta de dados é finalizado e a média de velocidade é calculada e todas as coordenadas, juntamente com o restante das informações, são enviados para o Firebase. Se o usuário não estiver conectado à internet, assim que ele ativar a rede móvel de seu dispositivo ou se conectar a uma rede wi-fi, os dados serão enviados para o banco de dados, concluindo assim o processo de captura das informações do percurso. 


\subsection{Website}

Para a construção do website, foram utilizadas as tecnologias: HTML5, CSS3, JavaScript e jQuery. $\mathrm{O}$ website possibilita que os usuários acompanhem no mapa em tempo real os veículos conectados com o aplicativo e fornece uma interface, possibilitando aos usuários que façam o download dos dados coletados para serem utilizados na simulação, podendo selecionar os dados desejados, informando a data de início e fim da pesquisa.

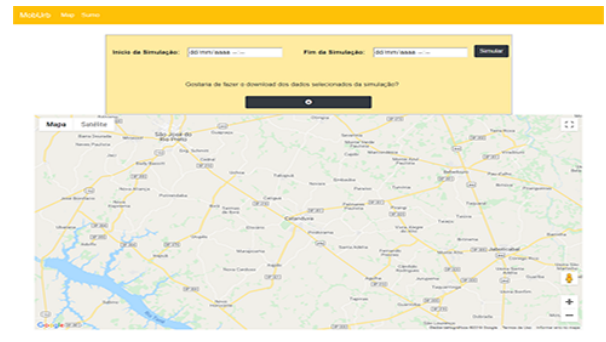

Figura 2. Website de Rastreamento e Download

\subsubsection{Geração do modelo de mobilidade para os simuladores de rede}

O SUMO (Simulador de Mobilidade Urbana) é um pacote de simulação de tráfego de código aberto, projetado para lidar com grandes redes rodoviárias. Por meio deste, é possível realizar diversos tipos de pesquisa, principalmente no contexto de gerenciamento de tráfego e mobilidade urbana. O SUMO permite gerar diferentes saídas para cada execução de simulação. Diversos dados podem ser definidos e extraídos por meio das simulações, tais como: informações de rotas para cada um dos veículos, medidas para ruas e faixas, consumo médio de combustível, emissão média de poluentes, entre outros [Daniel Krajzewicz and Bieker 2012].

Foi desenvolvido um algoritmo responsável por decodificar o banco de dados JSON, em linguagem de marcação XML, na estrutura padrão utilizada pelo SUMO, para que os dados coletados possam ser utilizados como base de simulações com dados reais das rodovias brasileiras. Estes dados passam por um processo de mapeamento, onde as informações dos percursos dos veículos, como tipo de veículo, data e hora e coordenadas são repassados para um arquivo XML interpretável pelo simulador descrito acima.

Para realizar a conversão das coordenadas coletadas para nós ou vértices, foi utilizado um módulo Python denominado Sumolib. Este módulo provê diversas funcionalidades para trabalhar com simulação. As funcionalidades utilizadas para gerar os arquivos de simulação foram: convertLonLatXY, responsável por realizar a conversão de coordenadas em pontos $(\mathrm{X}, \mathrm{Y})$, e getNeighboringEdges, responsável por encontrar os nós e/ou vértices próximos à posição encontrada. A Figura 3 representa a simulação do mapa da cidade de Catanduva (SP).

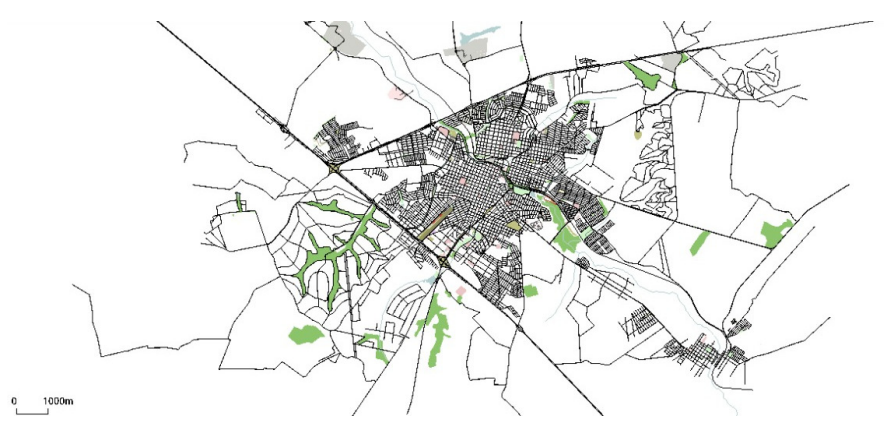

Figura 3. Simulação do mapa de Catanduva (SP) 


\subsubsection{Monitoramento de Veículos}

A construção do mapa de monitoramento do website foi feito através da API JavaScript do Google Maps, onde é possível que os usuários do website vejam todos os dispositivos conectados no momento. Para que um dispositivo fique visível no mapa de monitoramento, é necessário que o usuário do aplicativo móvel inicie o serviço de captura de coordenadas. O usuário do website pode filtrar o tipo de veículo que deseja visualizar no mapa por meio de uma caixa de seleção, onde estão disponíveis os tipos de veículos, que são: carros, motos, bicicletas, ônibus, caminhões e não informados. A Figura 4 mostra o website com o mapa e os veículos conectados.

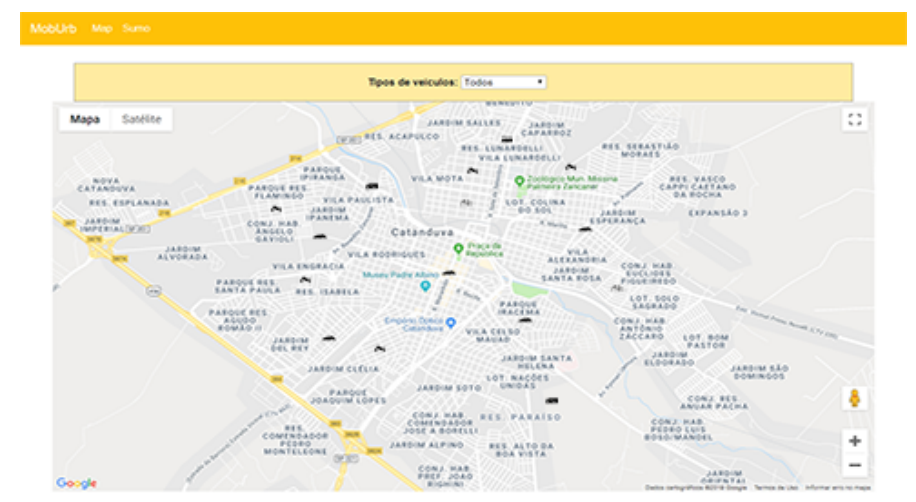

Figura 4. Website de Monitoramento

\subsection{Disponibilização do código, documentação e descriçao da apresentação}

Os componentes que compõem a ferramenta MobUrb foram desenvolvidos sob a licença GNU General Public License (Licença Pública Geral GNU), GNU GPL e encontra-se disponível no link: http://rsd.ctd.ifsp.edu.br/?page $i d=85$.

Nesse link encontram-se os seguintes dados:

- Fonte: links para o código fonte de cada componente da ferramenta, desde o aplicativo de sensoriamento, bem como o website.

- Site: link para o site de geração dos arquivos de simulação, bem como a interface de monitoramento de mobilidade.

- App: link para a instalação do aplicativo de sensoriamento.

- Documentação: consta um link para um tutorial de uso do aplicativo e do site, bem como o link para o vídeo explicativo, demonstrando a instalação e uso de todos os componentes da ferramenta.

Para a apresentação da ferramenta será necessária uma máquina com acesso à internet e as configurações do Sumo instaladas. A apresentação da ferramenta será iniciada com a descrição do aplicativo de sensoriamento, na sequência a explicação do website e a geração dos dados para a simulação do modelo de mobilidade para o Sumo.

\section{Avaliação do aplicativo de sensoriamento}

Para a avaliação de desempenho do aplicativo de sensoriamento, foram levados em consideração o consumo de bateria e dados dos planos de dados de telefonia (4G). Os testes foram realizados em três modelos diferentes de smartphones, ambos com o sistema operacional Android.

O primeiro smartphone (Smartphone 1) tem 64GB de memória de armazenamento, sistema operacional Android versão 8.1.0, display de 5.5 polegadas, dados móveis LTE 4G, rede GSM Quad Band (850/900/1800/1900), Chipset Cortex-A53 Qualcomm Snapdragon 625 MSM8953, processador de $2 \mathrm{GHz}$ com 8 núcleos (octa-core), sistema operacional de 64 bits, unidade de processamento gráfico Adreno 506, 4GB de memória 
RAM, wifi 802.11, velocidade máxima de download 150 Mbps velocidade de upload de 50 Mbps, possui uma bateria do tipo LiPo com capacidade de $3080 \mathrm{mAh}$.

O segundo smartphone (Smartphone 2) tem 32GB de memória de armazenamento, sistema operacional Android versão 8.1.0, display de 5 polegadas, dados móveis LTE 4G, rede GSM Quad Band (850/900/1800/1900), Chipset Qualcomm Snapdragon 430 (SDM430), processador de 8 núcleos (octa-core) 4x $1.4 \mathrm{GHz}$ Cortex-A53 e 4x $1.1 \mathrm{GHz}$ Cortex-A53, sistema operacional de 64 bits, unidade de processamento gráfico Adreno 505, 2GB de memória RAM, wifi 802.11, velocidade máxima de download $150 \mathrm{Mbps}$, velocidade de upload50 Mbps, possui uma bateria do tipo Litio com capacidade de 2800 mAh.

O terceiro smartphone (Smartphone 3) tem 64GB de memória de armazenamento, sistema operacional Android versão 8.0.0, display de 5.5 polegadas, dados móveis LTE 4G, rede GSM Quad Band (850/900/1800/1900), Chipset Cortex-A53 Qualcomm Snapdragon 630, processador de 8 núcleos (octa-core) 4x 2.2 GHz Cortex-A53 + 4x $1.8 \mathrm{GHz}$, sistema opepracional 64bits, unidade de processamento gráfico Adreno 508, 4GB de memória RAM, wifi 802.11, velocidade máxima de download $150 \mathrm{Mbps}$, velocidade de upload50 Mbps, possui uma bateria do tipo Litio com capacidade de $3300 \mathrm{mAh}$.

A figura 5(a) mostra a quantidade de dados móveis utilizados durante a execução. O consumo de dados móveis durante a execução do aplicativo é considerado baixo devido ao maior consumo (Smartphone 1) ter utilizado apenas $63,12 \mathrm{~KB}$ e o menor consumo ter utilizado 54,76 KB pelo Smartphone 2. O baixo consumo de dados móveis se dá devido ao aplicativo enviar as coordenadas do usuário a cada dois minutos e enviar todas as informações da rota apenas uma vez, somente quando a rota for concluída. O Smartphone 1 teve um consumo de dados móveis um pouco maior em comparação aos demais smartphones(2 e 3). Isto aconteceu porque a rota tomada pelo usuário do Smartphone 1 foi maior que a utilizada pelos outros usuários; o que influencia diretamente a quantidade de coordenadas e de dados enviados.

Figura 5(b) mostra o consumo da bateria. Este consumo é considerado baixo, pois o Smartphone 3 alcançou somente $1 \%$ do consumo de bateria em vinte minutos de teste (23mAh), e nos Smartphones 1 e 2, o gasto não atingiu $1 \%$ (8 e $10 \mathrm{mAh}$ ). $\mathrm{O}$ baixo consumo de bateria ocorre devido à execução do aplicativo em segundo plano, permitindo que o usuário desligue a tela do smartphone. O usuário do Smartphone 1 tinha um consumo de bateria menor do que o do Smartphone 3. Isto acontece devido ao uso de outros aplicativos simultâneamente durante a execução do aplicativo de sensoriamento, o que pode influenciar no consumo da bateria.

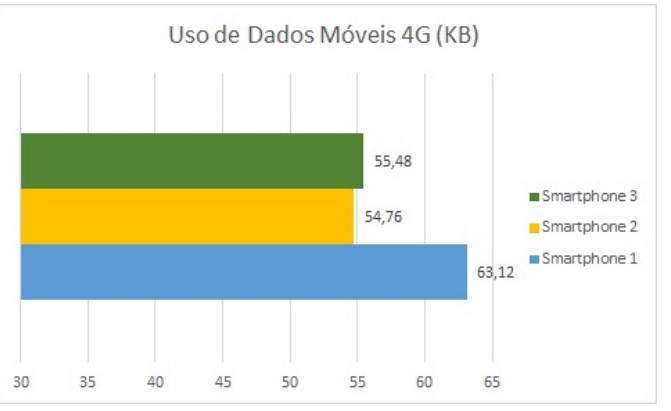

(a) Média de consumo de dados móveis

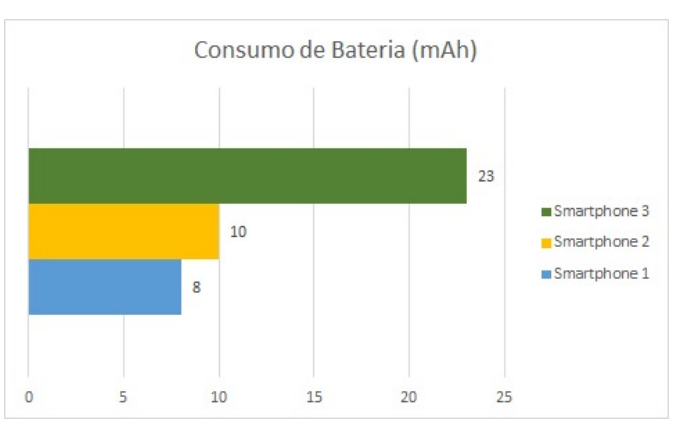

(b) Média de consumo de bateria

Figura 5. Avaliação do aplicativo de Sensoriamento

\section{Conclusão}

Este artigo apresenta o desenvolvimento e as funcionalidades de uma ferramenta utilizada para o monitoramento de veículos e geração de dados em tempo real da mobilidade urbana da cidade de Catanduva (SP). A ferramenta consiste em um aplicativo móvel, responsável por capturar as coordenadas geográficas dos usuários de maneira anônima, consumindo 
poucos recursos do smartphone e realiza o armazenamento das informações referentes às rotas reais, no banco de dados, que podem ser utilizadas em simuladores de redes. Foi realizada uma avaliação simples com relação ao aplicativo de sensoriamento, na qual os resultados mostraram que o aplicativo possui baixo consumo de dados móveis e de bateria.

Como trabalhos futuros, pretende-se fazer novos serviços de sistemas de transportes inteligentes baseados nas informações coletadas por esse aplicativo.

\section{Agradecimentos}

Rodolfo I. Meneguette e agradece a CNPq (processos 150545/2018-5, 407248/2018-8) por financiar seu projeto de pesquisa.

\section{Referências}

Boukerche, A. and Meneguette, R. I. (2017). Vehicular cloud network: A new challenge for resource management based systems. In 2017 13th International Wireless Communications and Mobile Computing Conference (IWCMC), pages 159-164.

Bracciale, L., Bonola, M., Loreti, P., Bianchi, G., Amici, R., and Rabuffi, A. (2014). Crawdad dataset roma/taxi (v. 2014-07-17). See http://crawdad. org/roma/taxi/20140717.

Codeca, L., Frank, R., and Engel, T. (2015). Luxembourg sumo traffic (lust) scenario: 24 hours of mobility for vehicular networking research. In 2015 IEEE Vehicular Networking Conference (VNC), pages 1-8.

Daniel Krajzewicz, Jakob Erdmann, M. B. and Bieker, L. (2012). Recent development and applications of sumo - simulation of urban mobility. International Journal On Advances in Systems and Measurements, 5:128-138.

Grzybek, A., Seredynski, M., Danoy, G., and Bouvry, P. (2012). Aspects and trends in realistic vanet simulations. In 2012 IEEE International Symposium on a World of Wireless, Mobile and Multimedia Networks (WoWMoM), pages 1-6. IEEE.

Harri, J., Filali, F., and Bonnet, C. (2009). Mobility models for vehicular ad hoc networks: a survey and taxonomy. IEEE Communications Surveys Tutorials, 11(4):19-41.

Huang, H., Luo, P., Li, M., Li, D., Li, X., Shu, W., and Wu, M. (2007). Performance evaluation of suvnet with real-time traffic data. IEEE Transactions on Vehicular Technology, 56(6):3381-3396.

Huang, H., Zhang, D., Zhu, Y., Li, M., and Wu, M.-Y. (2012). A metropolitan taxi mobility model from real gps traces. J. UCS, 18(9):1072-1092.

I. Meneguette, R., E. De Grande, R., and A. F. Loureiro, A. (2018). Implementation and Testing Tools, pages 167-182. Springer International Publishing, Cham.

Kuran, M. , Carneiro Viana, A., Iannone, L., Kofman, D., Mermoud, G., and Vasseur, J. P. (2015). A smart parking lot management system for scheduling the recharging of electric vehicles. IEEE Transactions on Smart Grid, 6(6):2942-2953.

Meneguette, R. I. and Boukerche, A. (2017). Peer-to-peer protocol for allocated resources in vehicular cloud based on v2v communication. In 2017 IEEE Wireless Communications and Networking Conference (WCNC), pages 1-6.

Piorkowski, M., Sarafijanovic-Djukic, N., and Grossglauser, M. (2009). Crawdad data set epfl/mobility (v. 2009-02-24).

Uppoor, S., Trullols-Cruces, O., Fiore, M., and Barcelo-Ordinas, J. M. (2014). Generation and analysis of a large-scale urban vehicular mobility dataset. IEEE Transactions on Mobile Computing, 13(5):1061-1075.

Wang, S., Djahel, S., and McManis, J. (2015). An adaptive and vanets-based next road re-routing system for unexpected urban traffic congestion avoidance. In 2015 IEEE Vehicular Networking Conference (VNC), pages 196-203. 